\title{
BILATERAL PAPILLOEDEMA IN A CASE OF REACTIVATED BREAST CARCINOMA SECONDARY TO IDIOPATHIC INTRACRANIAL HYPERTENSION
}

Wasea Narmawala ${ }^{1}$, Devendra Saxena ${ }^{2}$

\section{HOW TO CITE THIS ARTICLE:}

Wasea Narmawala, Devendra Saxena. "Bilateral Papilloedema in a Case of Reactivated Breast Carcinoma Secondary to Idiopathic Intracranial Hypertension". Journal of Evolution of Medical and Dental Sciences 2014; Vol. 3, Issue 66, December 01; Page: 14421-14423, D0I: 10.14260/jemds/2014/3934

INTRODUCTION: A 48 years old female was referred with blurring of vision in OU since 10 days. She was operated for breast carcinoma 3 years back with completion of one cycle of Radiotherapy and Chemotherapy. She was diagnosed with reactivation of metastasis in liver and stomach for which she was undergoing CT (6 cycles April-July). BCVA was 6/12 and N6 in OU. Colour vision and contrast sensitivity were normal. On examination pupils central \& were reacting to light. Slit lamp showed no iris seeding, no NVI and quiet anterior segment. Applanation tensions of both eyes were $14 \mathrm{~mm} \mathrm{Hg}$. Fundus showed OU Marked Disc edema with peripapillary haemorrhages and retinochoroidal folds at macula. Patient was started on systemic steroids. B scan and MRI orbit was advised to rule out Optic nerve head metastasis, choroidal metastasis and optic nerve drusen- which were negative and CSF study was normal. 1 week later, BCVA improved 6/9 in OU while fundus showed OU marked disc edema with choroiretinal folds (OD patons folds at macula) with Breakthrough Hemorrhage in vitreous in OD. High dose steroids, acetozalamide and aspirin were started and BCVA improved 6/6 in 2 weeks with reappearance of spontaneous venous pulsations. After improvement of vision, steroids were tapered and dose of acetozalamide was decreased. 2 weeks later, patient came with blurring of vision with headache and tinnitus. BCVA was reduced to 6/9 in OU. Fundus showed marked increase in the disc edema. Neurosurgery reference was done for surgical options: optic nerve sheath decompression and fenestration or shunt surgery.

\section{DISCUSSION:}

- Idiopathic intracranial hypertension is a diagnosis of exclusion.

- It is characterized by rise of intracranial pressure without any mass lesions in the brain.

- Mechanisms include: increased CSF production, increase in volume of brain or blood (Secondary to increased viscosity of blood) or obstruction to the venous flow of the brain.

- Various diagnostic criterias are available to diagnose this condition.(1)

- Normal MRI/CT with normal CSF analysis with high opening CSF pressure $>25 \mathrm{~cm} \mathrm{H2O} \mathrm{in}$ presence of signs and symptoms of raised intracranial pressure and no mass lesions are points to be considered. (Modified Dandy's criteria).(1)

- Control of intracranial pressure is done with medical therapy like carbonic anhydrase inhibitors, antiplatelets and high dose steroids.

- In this case, there was a gradual increase in visual acuity with decrease in disc edema with medical therapy but due to venous stasis there was breakthrough hemorrhage into the vitreous cavity.

- For this case, there was a need for surgical intervention because patient was intolerant to maximum medical therapy with reactivation of symptoms on decreasing the dose

- Choice of surgery: shunt surgery to relieve patient of tinnitus, headache along with visual symptoms.(2) 


\section{CASE REPORT}

\section{REFERENCES:}

1. Michael Wall, M.D, Idiopathic Intracranial Hypertension, Neurol Clin. Aug 2010; 28 (3): 593617.

2. Banta JT ${ }^{1}$, Farris BK, Pseudotumor cerebri and optic nerve sheath decompression, Ophthalmology. 2000 0ct; 107 (10): 1907-12.

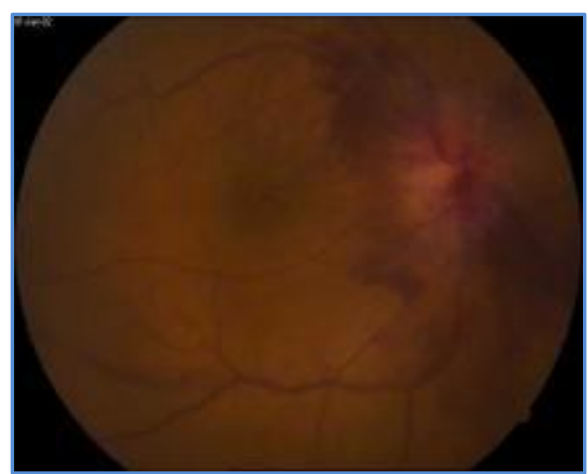

OD- Disc edema

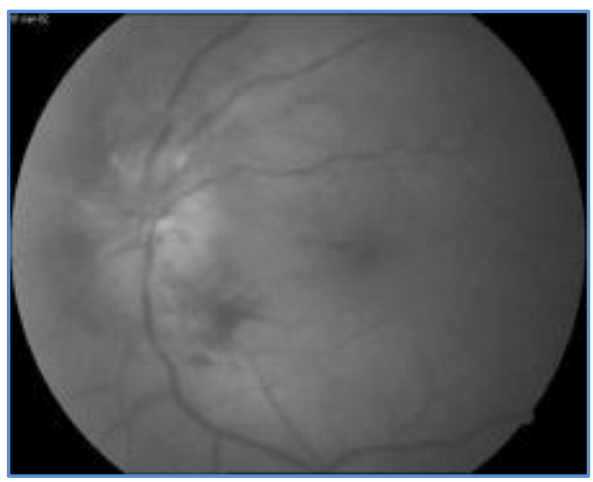

OD-red free

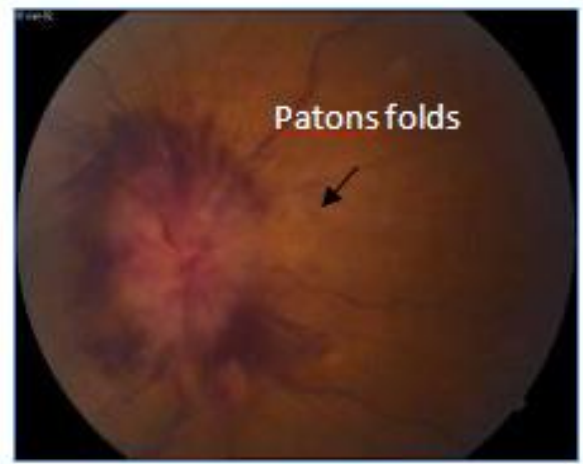

OD-breakthrough hemorrhage

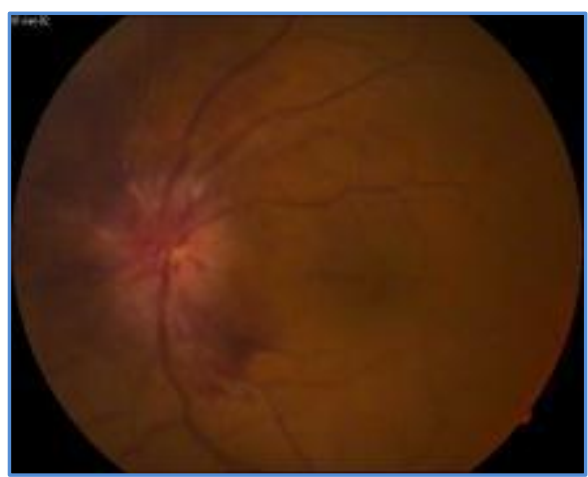

OS-Disc edema

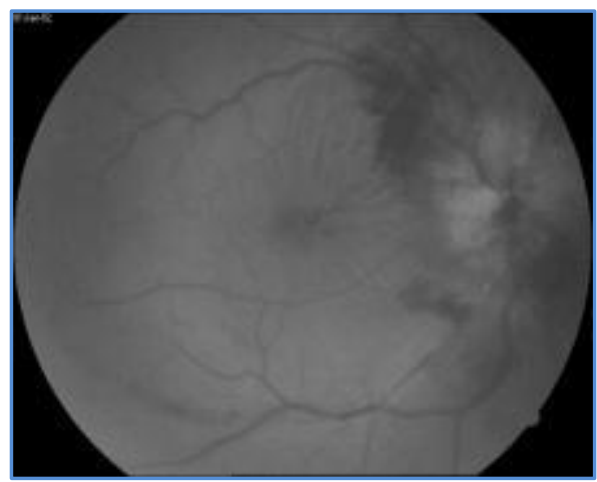

OS - red free

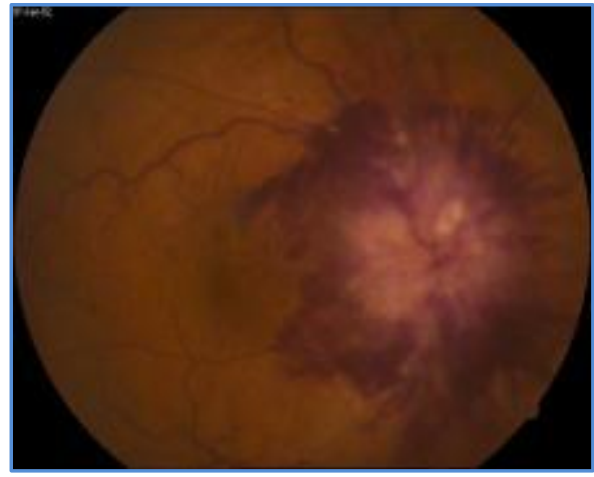

OS-breakthrough hemorrhage 


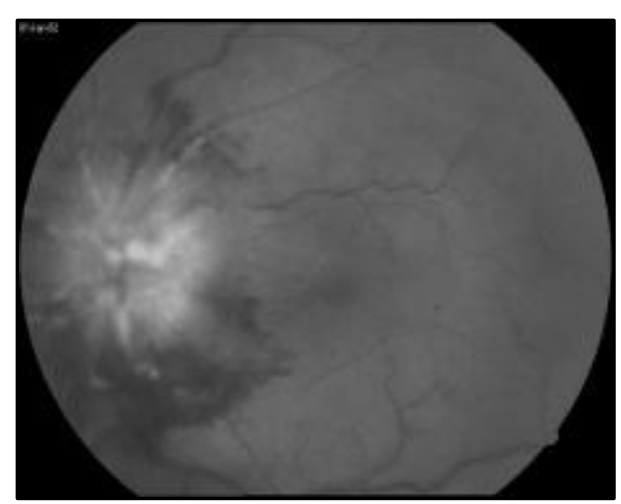

OD red free

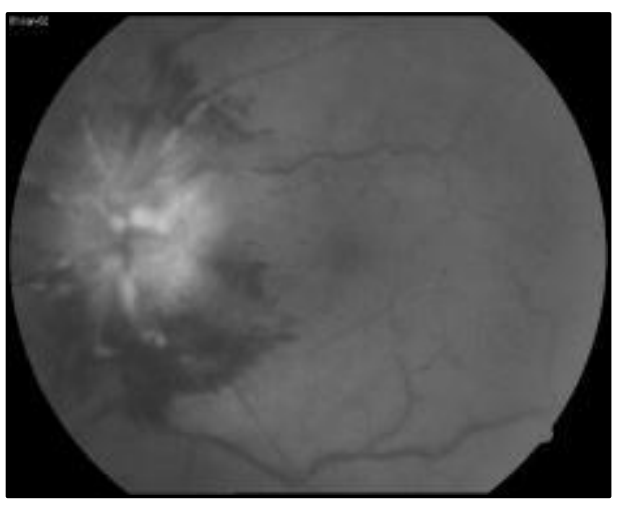

OS red free

\section{AUTHORS:}

1. Wasea Narmawala

2. Devendra Saxena

\section{PARTICULARS OF CONTRIBUTORS:}

1. Second year Resident, Department of Ophthalmology, Shree Krishna Hospital.

2. Associate Professor, Department of Ophthalmology, Shree Krishna Hospital.

\section{NAME ADDRESS EMAIL ID OF THE CORRESPONDING AUTHOR:}

Dr. Wasea Narmawala, \# 201, Eye OPD,

Shree Krishna Hospital, Gokul Nagar,

Karamsad, Anand-388325.

Email: drwasea@gmail.com

Date of Submission: 31/10/2014. Date of Peer Review: 03/11/2014. Date of Acceptance: 27/11/2014. Date of Publishing: 01/12/2014. 